\title{
Early Morning Functioning in Stimulant-Treated Children and Adolescents with Attention-Deficit/Hyperactivity Disorder, and its Impact on Caregivers
}

\author{
Floyd R. Sallee, MD, PhD
}

\begin{abstract}
Objective: The purpose of this study was to examine the temporal occurrence and severity of inadequate attention-deficit/ hyperactivity disorder (ADHD) symptom control throughout the day, and, more specifically, the frequency and severity of associated functional impairments and their apparent emotional impact on parents and caregivers during the early morning routine before school, in children and adolescents with ADHD currently treated with stable doses of stimulant medications. Methods: Information was obtained from 201 primary caregivers of children and adolescents with ADHD using a selfadministered, on-line quantitative research survey.

Results: Inadequately controlled ADHD symptoms were rated as most severe during the evening homework time and the early morning routine. The majority of caregivers reported early morning ADHD symptoms and impairment of early morning functioning (EMF) as moderate to severe. Caregiver reactions to their child's early morning ADHD symptoms and unwanted behaviors included feeling overwhelmed, exhausted, and constantly stressed.

Conclusions: Control of EMF impairments from inadequately controlled ADHD symptoms is a significant unmet need in children and adolescents with ADHD treated with stable morning doses of stimulant medications. Current orally administered stimulant treatment options have not addressed this challenge.
\end{abstract}

\section{Introduction}

A TTENTION-DEFICIT/HYPERACTIVITY DISORDER (ADHD) is among the most common childhood psychiatric conditions (Pliszka and AACAP Work Group on Quality Issues 2007) with an estimated prevalence range of $5.9-7.1 \%$ in children and adolescents (Willcutt 2012). According to the Diagnostic and Statistical Manual of Mental Disorders, 5th ed. (DSM-V), the essential diagnostic feature of ADHD is a persistent pattern of inattention and/ or hyperactivity/impulsivity that interferes with functioning or development (American Psychiatric Association 2013). In order to meet the diagnostic criteria for ADHD, there must be clear evidence of interference with, or the reduced quality of, social, academic, or occupational functioning attributable to the symptoms (American Psychiatric Association 2013). A host of studies have associated ADHD in children and adolescents with significant behavioral consequences including psychopathology, school and occupational failure, family and peer difficulties, emotional problems, and low self-esteem (Shaw et al. 2012). Additionally, ADHD has also been shown to have negative effects on families as a whole, such as disturbed interpersonal relationships, less perceived family cohesive- ness and greater conflict, depression in parents, and higher incidences of divorce and separation (Wymbs et al. 2008; Chang et al. 2013; Gau and Chang 2013). Accordingly, the American Academy of Child and Adolescent Psychiatry Practice Parameter for the Assessment and Treatment of Children and Adolescents With Attention-Deficit/Hyperactivity Disorder recommends that a wellthought-out and comprehensive treatment plan for patients with ADHD should consist of psychopharmacologic and/or behavior therapy (Pliszka and AACAP Work Group on Quality Issues 2007).

Behavioral symptoms in children with ADHD have been shown to fluctuate across the day (Antrop et al. 2005). Stimulant-based medications such as methylphenidate (MPH) and amphetamines (AMP) have been the cornerstone of ADHD pharmacotherapy for several decades (Pliszka and AACAP Work Group on Quality Issues 2007), and their temporal effects on behavioral symptoms in children and adolescents have been assessed at different times throughout the day. The earliest temporal assessments of stimulant treatment effect focused on adequate control of ADHD symptoms in school. In the last decade, the temporal effects of stimulant medications in controlling ADHD symptoms have evolved in parallel with the evolution of numerous long-acting, extended-

Department of Psychiatry \& Behavioral Neuroscience, University of Cincinnati College of Medicine, Cincinnati, Ohio.

Funding: This study was funded by Ironshore Pharmaceuticals \& Development, Inc.

(c) The Author(s) 2015; Published by Mary Ann Liebert, Inc. This Open Access article is distributed under the terms of the Creative Commons Attribution Noncommercial License (http://creativecommons.org/licenses/by-nc/4.0/) which permits any noncommercial use, distribution, and reproduction in any medium, provided the original author(s) and the source are credited. 
release (ER) stimulant medications; to include both in-school and after-school symptom control.

Although it is known that many home-based difficulties for children and adolescents with ADHD occur before the school day begins (Whalen et al. 2006) and that the time from awakening to arrival at school may represent up to $20 \%$ of the day ( $\sim 2-3$ hours) for children and adolescents with ADHD and their families (Wilens et al. 2010), little is known about the specific nature of early morning functioning (EMF) impairments in stimulant-treated children and adolescents with ADHD or the impact of these impairments on caregivers.

Recent interest has now begun to focus on the issue of inadequately controlled ADHD symptoms that occur during the early morning hours before the school day begins and to date, three pharmacotherapy-based efficacy studies have addressed the effects of different MPH formulations on EMF in children and adolescents with ADHD (Sangal et al. 2006; Wilens et al. 2010; Döpfner et al. 2011). The earliest of the published studies was a randomized, double-blind, crossover trial designed to assess the effects of threetimes-daily administration of immediate-release (IR) MPH (before 8:00 a.m., at or near noon, and between 4:00 and 5:00 p.m.) versus twice daily atomoxetine on sleep parameters in 85 children and adolescents ages 6-14 with ADHD (Sangal et al. 2006). The 13 item Daily Parent Rating of Evening and Morning Behavior (DPREMB) scale was among the study's secondary outcome measures and included caregiver ratings of core ADHD symptoms and behavioral problems typically experienced by children with ADHD in the early morning (4 items) and late afternoon/evening (9 items) (Michelson et al. 2002). The results of the study pertaining to improvements in EMF demonstrated that atomoxetine was shown to have significant beneficial treatment effects on two of the four EMF items as compared to the thrice-daily IR MPH regimen. The mean changes from baseline on the DPREMB for the morning subscale score were -1.18 for atomoxetine and -0.41 for IR MPH, which was statistically significant favoring atomoxetine $(p<0.001)$. When looking specifically at the treatment effect for the IR-MPH group on the DPREMB (morning) parent-rated questions, "How difficult was it to get your child out of bed this morning?", "How much difficulty did your child have getting ready this morning?", "How much was your child arguing or struggling excessively this morning?", and "How irritable and quick was your child to lose his or her temper this morning?", the mean change scores compared with baseline values ranged from 0.03 (worsening) to -0.22 (minor improvement). In summary, oral administration of IR-MPH before 8:00 a.m. did not demonstrate statistically significant improvements in early morning ADHD symptoms and behaviors in children and adolescents when compared with baseline values (Sangal et al. 2006).

The second study (Döpfner et al. 2011), was a non-interventional, non-controlled, multicenter, prospective, observational, postmarketing surveillance study, which assessed the efficacy of a once-daily modified-release MPH formulation in 822 children and adolescents with ADHD ages 6-17 years. Although the time of dosing was not specified, most once-daily ADHD medications are administered in the morning immediately after awakening or at breakfast time. As a secondary measure, this study used the parent version of the Day Profile of ADHD Symptoms (DAYAS-P) questionnaire, a new rating scale that assesses the daily profile of ADHD externalizing symptoms from early morning until bedtime (Breuer et al. 2011). Data from the DAYAS-P questionnaire indicated that treatment resulted in statistically significant improvement $(p<0.001)$ in the total score for morning/before-school symptoms and behavioral problems from visit $1(1.37 \pm 0.77)$ to visit $3(1.02 \pm 0.72)$, and for the ADHD symptom score for the morning before school from visit 1 $(1.38 \pm 0.71)$ to visit $3(1.02 \pm 0.78)$, although the Cohen's $d$ for effect size for both ratings was rather low ( 0.51 and 0.47 , respectively) (Döpfner et al. 2011).

Only one randomized, placebo-controlled study has focused exclusively on inadequately controlled ADHD symptoms and associated EMF impairments in children ages 6-12 years with ADHD by examining the efficacy of a transdermal MPH formulation (MTS) administered once-daily between 6:00 and 7:00 a.m. (Wilens et al. 2010). The study's primary outcome measures include the ADHD-Rating Scale (ADHD-RS) and ADHD-AM-RS (for the time period 6:00-9:00 a.m. only). This study also included a new clinician-rated and completed 20 item questionnaire (the BeforeSchool Functioning Questionnaire [BSFQ]) as a secondary outcome measure. The questionnaire was generated from commonly reported areas of dysfunction in the early morning (6:00-9:00 a.m.), before-school activities associated with ADHD symptomatology, such as breakfast, hygiene, time awareness, and getting to school. The psychometric properties of the BSFQ have recently been validated by Faraone et al. (2015). Results of the study demonstrated significant reductions with MTS compared to placebo for the ADHD-RS total score $(p<0.001)$, ADHD-AM-RS $(p=0.003)$, and BSFQ total score $(p=0.001)$. Of the 20 before-school items that comprised the BSFQ, only three did not show statistically significant differences $(p<0.05)$ between the transdermal MPH- and placebo-treated groups ("Did your child have difficulty with: being quiet; awaiting his/her turn; and getting to school?"). There was a $69 \%$ reduction in the subjects' total score for transdermal MPH versus a $23 \%$ reduction for transdermal placebo. The items that had the largest difference in favor of transdermal MPH versus placebo included listening, following directions, attention, distraction, and hygiene $(p<0.01)$ (Wilens et al. 2010).

When viewed collectively, the results from the two aforementioned pharmacologic studies with orally administered MPH suggesting that the acute treatment has benefits for inadequately controlled early morning ADHD symptoms and associated behaviors are inconclusive. These mixed results also suggest the need for further research on EMF impairments in stimulant-treated children and adolescents with ADHD. Further, these three studies appear to suggest a need for a more formalized assessment of EMF, as well as a need for further exploration regarding the mechanisms by which pharmacotherapies may improve some aspects of early morning ADHD symptoms and functioning, as opposed to others.

In addition to pharmacotherapeutic efficacy studies, one large, on-line, parent-completed questionnaire survey study conducted in Europe was designed to examine the impact of ADHD on their children's/adolescent's everyday activities, general behavior, and family relationships, and to assess the effect of stimulant medications on the behaviors of their children and adolescents with ADHD during times of day when parents have the closest contact with their children and adolescents, such as the early morning (Coghill et al. 2008). Survey results demonstrated that a significantly higher percentage of children and adolescents ages 6-18 years with ADHD receiving stimulant medication experienced impaired functioning during their morning routine (from 7:00 to 8:00 a.m.; waking up, getting ready for school) compared with unmedicated children and adolescents with ADHD (55\% vs. $36 \%$, respectively; $p<0.05$ ). Additionally, parents reported that children and adolescents with ADHD receiving 12 hour stimulant medications experienced greater levels of functional impairments during the morning routine than those receiving 6-8 hour stimulant medications. Coghill et al. noted a possible limitation of the study in that traditionally in 
Europe, the most severely symptomatic and impaired ADHD children and adolescents are the ones who are treated with stimulant medications (in general) and further, that longer acting stimulant medications (12 hour) are often reserved for the most severely ill patients whereas the 6-8 hour medications are typically utilized for less severely ill ADHD patients. Therefore, this stratification of ADHD patient symptom severity assignment to stimulant treatment and use of modified-release formulations specifically for the most highly symptomatic patients, may account for the apparent diminished benefit of stimulant treatment on early morning ADHD symptoms reported by the authors. Importantly, this large quantitative research survey study clearly detected evidence of early morning ADHD symptomatology and related EMF impairments in children and adolescents treated with stimulant medications. In aggregate, the survey's EMF findings add further evidence to pharmacologic studies in suggesting that morning administration of oral stimulant medications may not provide meaningful clinical control of early morning ADHD symptoms, so as to improve functioning during the school-age child's early morning routines (Coghill et al. 2008).

In summary, a review of the current ADHD clinical literature would suggest that despite recent formulation advances in oral stimulant medications designed to provide increasingly longer duration of treatment benefit throughout the day, very little is known about the specific prevalence, frequency, and severity of inadequately controlled ADHD symptoms and related EMF impairments that likely occur during the early morning routine in stimulant-treated children and adolescents.

The objectives of this parent self-report-based, quantitative research survey were to determine the temporal occurrence and severity of inadequately controlled ADHD symptoms throughout the day in children and adolescents with ADHD who are currently treated with stable doses of stimulant medications. More importantly, the survey allowed for a quantitative exploration of the temporal occurrence, frequency, and severity of inadequately controlled ADHD symptoms specifically related to the early morning hours of the day, before school; as well as detailed descriptions of related functional impairments, unwanted behaviors and their apparent emotional impact on parents and caregivers.

\section{Methods}

\section{Survey development and description}

This self-administered, anonymous, on-line quantitative research survey was conducted between December 31, 2012 and January 24 , 2013. An on-line, primary caregiver-completed questionnaire was designed with input from experts in the field of child and adolescent psychiatry to determine from parent self-reports if inadequately controlled ADHD symptoms exist in children and adolescents with ADHD who are currently treated with stable doses of stimulant medications. Second, parents were asked to define these inadequately controlled ADHD symptoms, if present, with respect to their temporal occurrence and severity. Of particular interest were the detailed responses of those parents who identify the "Early Morning Routine," defined in the survey as "from the moment the child/adolescent awakens to the time they leave for school" and including activities such as getting out of bed, getting dressed, brushing teeth, sitting down for breakfast, having breakfast, and getting ready to leave the household, as a problematic time period for the inadequate control of ADHD symptoms. Caregivers who identified inadequately controlled ADHD symptoms during the Early Morning Routine (Likert severity rating $>2$ ) were asked to continue the survey by answering a series of multiple choice and open-ended questions. This portion of the survey allowed respondents to provide detailed descriptions of related functional mpairments and unwanted behaviors that manifest from these inadequately controlled ADHD symptoms. Additionally, it allowed for assessment of their ADHD symptom severity and frequency of early morning ADHD symptom occurrence across the weekdays when school was in session.

The survey contained 32 primary questions and 30 subquestions for a total of 62 questions. On average, the time taken for primary caregivers of children/adolescents with ADHD to complete the survey was 20 minutes.

This study was sponsored by Ironshore Pharmaceuticals \& Development, Inc. and conducted by Repass \& Partners, an experienced market research firm. The survey did not contain any option for any of the 18 standard types of protected health information (PHI) data to be collected. Surveys were completed voluntarily and anonymously. Caregiver respondents were blinded as to the research sponsor, and the survey was conducted in accordance with and adherence to The Marketing Research Association's (MRA) Code of Marketing Research Standards (Marketing Research Organization 2013).

\section{Sampling strategy}

Parent/primary caregiver research respondents were derived from nationally representative consumer research panels consisting of both presumed or known households of children and adolescents with ADHD $(n=10,750)$. To ensure that 200 surveys were completed, an additional invitation was sent to households with unknown child/adolescent-ADHD diagnostic status $(n=142,999)$. Primary caregivers who volunteered to participate in the survey first completed a screening questionnaire to ensure that the following requisite inclusion criteria were met prior to beginning the general survey: 1) Must be the parent ( $\geq 65 \%$ of completed surveys) or primary caregiver for a child/adolescent 6-17 years of age diagnosed with ADHD and 2) that child or adolescent's primary ADHD medication must be a stimulant medication and 3) the dosage of the stimulant medication must have been stable for at least 3 months prior to taking the survey. Caregivers who were employed by a physician, a hospital, a pharmaceutical manufacturer, or a pharmacy or marketing research firm were not eligible to participate.

Eligible respondents were subsequently screened to assess for the presence of inadequately controlled ADHD symptoms at any time throughout the day. If inadequately controlled ADHD symptoms were present, the caregiver was asked to identify in which of the following temporal periods of the day did the symptoms manifest and at what severity level (Likert scale 1-10): 1) The Early Morning Routine, 2) During the School Day, 3) Afternoon Homework Time, 4) Dinner Time, 5) Evening Homework Time, and 6) Bedtime.

\section{Results}

A total of 2013 caregivers volunteered to participate in the survey and $290(14.0 \%)$ met the requisite screening criteria and entered into the formal survey; 280 respondents (97.0\%) identified at least one temporal period of the day when their child's/adolescent's ADHD symptoms were inadequately controlled and 264 caregivers $(91.0 \%)$ specifically identified the Early Morning Routine as a time period when inadequately controlled ADHD 
symptoms manifested with at least mild severity (Likert scale $\geq 2$ ). A total of 201 caregivers completed the entire survey, including the specific details for how the Early Morning Routine was impacted by these inadequately controlled ADHD symptoms; therefore, this is the number that was used for the majority of the analyses.

Adult respondent and child/adolescent patient demographic information is presented in Tables 1 and 2, respectively. Among respondents, $45.3 \%$ (91/201) were between the ages of 36 and 45 , $33.3 \%(67 / 201)$ had completed college, $25.9 \%(52 / 201)$ reported earning \$50,000-75,000 per year, and 72.6\% (146/201) reported being the mother of a child/adolescent with ADHD. Almost half $(46.3 \%, 93 / 201)$ reported that there were two children/adolescents living in the household, and $50.1 \%(236 / 471)$ reported that the age of all children/adolescents living in the household was between 6 and 12 years and that $55.0 \%(259 / 471)$ of all the children/adoles-

Table 1. Adult Respondent Demographic Information

\begin{tabular}{|c|c|}
\hline & $\mathrm{n}(\%)$ \\
\hline \multicolumn{2}{|l|}{ Age of respondent (years) } \\
\hline $18-25$ & 1/201 (0.5) \\
\hline $26-35$ & $62 / 201(30.8)$ \\
\hline $36-45$ & $91 / 201(45.3)$ \\
\hline $46-55$ & 40/201 (19.9) \\
\hline $56-65$ & $7 / 201(3.5)$ \\
\hline \multicolumn{2}{|l|}{ Education level of respondent } \\
\hline High school & $31 / 201(15.4)$ \\
\hline Trade/Technical school & $12 / 201(6.0)$ \\
\hline Some college & $63 / 201(31.3)$ \\
\hline College graduate & $67 / 201(33.3)$ \\
\hline Masters/Advanced degree & 28/201 (13.9) \\
\hline \multicolumn{2}{|l|}{ Income level of respondent } \\
\hline$<\$ 30,000$ & 35/201 (17.4) \\
\hline$\$ 30,000-\$ 49,999$ & $45 / 201(22.4)$ \\
\hline$\$ 50,000-\$ 74,999$ & $52 / 201(25.9)$ \\
\hline$\$ 75,000-\$ 99,999$ & $32 / 201(15.9)$ \\
\hline$\$ 100,000-\$ 124,999$ & $17 / 201(8.5)$ \\
\hline$\geq \$ 125,000$ & $19 / 201(9.5)$ \\
\hline Refused to answer/Did not know & $1 / 201(0.5)$ \\
\hline \multicolumn{2}{|c|}{ Respondent relationship to child/adolescent with ADHD } \\
\hline Mother & $146 / 201(72.6)$ \\
\hline Father & $36 / 201(17.9)$ \\
\hline Other & $19 / 201(9.5)$ \\
\hline \multicolumn{2}{|c|}{ Total number of children/adolescents in the household } \\
\hline 1 & $45 / 201(22.4)$ \\
\hline 2 & $93 / 201(46.3)$ \\
\hline 3 & $32 / 201(15.9)$ \\
\hline 4 & $19 / 201(9.5)$ \\
\hline$\geq 5$ & $12 / 201(6.0)$ \\
\hline \multicolumn{2}{|c|}{ Age of all children/adolescents in the household (years) } \\
\hline$<6$ & $74 / 471(15.7)$ \\
\hline $6-12$ & $236 / 471(50.1)$ \\
\hline $13-17$ & $161 / 471(34.2)$ \\
\hline \multicolumn{2}{|c|}{ Gender of all children/adolescents in the household } \\
\hline Male & $259 / 471(55.0)$ \\
\hline Female & $212 / 471(45.0)$ \\
\hline \multicolumn{2}{|c|}{$\begin{array}{l}\text { Number of children/adolescents in the household taking ADHI } \\
\text { medication }\end{array}$} \\
\hline 1 & $175 / 201(87.1)$ \\
\hline 2 & $24 / 201(11.9)$ \\
\hline 3 & $2 / 201(1.0)$ \\
\hline
\end{tabular}

ADHD, attention-deficit/hyperactivity disorder. cents living in the household were male. The majority $(87.1 \%, 175 /$ 201) reported that only one child/adolescent in the household was receiving stimulant treatment for ADHD. As for the child/adolescent patients reported on by caregivers in completed surveys, $71.1 \%$ (143/201) were male, $59.7 \%(120 / 201)$ were 6-12 years of age, and the mean age was 11.3 years. The majority had been diagnosed with the combined subtype of ADHD (78.6\%, 158/201) and smaller numbers were diagnosed with the inattentive $(12.9 \%$, $26 / 201)$ and hyperactive-impulsive $(8.5 \%, 17 / 201)$ subtypes. Two thirds $(67.0 \%, 135 / 201)$ of the children and adolescents reported on by their caregivers had been diagnosed with one or more of nine comorbidities examined. The most frequently diagnosed comorbid conditions for these children and adolescents with ADHD were learning disability $(37.8 \%, 76 / 201)$, anxiety $(31.3 \%, 63 / 201)$, and oppositional defiant disorder (ODD) $(24.4 \%$, 49/201). Subgroup analyses of caregiver respondent ratings indicated that early morning ADHD symptom scores were higher for Medicaid patients than for those with commercial insurance, for children (6-12) than for adolescents (13-17), for children diagnosed with comorbid ODD than for those without, and for children taking a supplemental ADHD medication in addition to their primary stimulant medication than for those who were not.

MPH $\mathrm{HCl}$ ER was the most frequently reported primary ADHD medication $(30.3 \%, 61 / 201)$, and $59.7 \%(120 / 201)$ of these children/adolescents with ADHD were not receiving any supplemental ADHD medications. Of those who did receive supplemental ADHD medications, AMP and dextroamphetamine mixed salts, IR (9.0\%, 18/201), MPH HCl IR (7.0\%, 14/201), and MPH HCl ER $(7.0 \%, 14 / 201)$ were the most frequently used. Almost one third of subjects were reported to have been taking their primary ADHD medication for 6-12 months $(30.8 \%, 62 / 201)$, and an equal number had been receiving their primary ADHD medication for $>2$ years. Approximately two thirds of the patients $(67.2 \%, 135 / 201)$ received their primary ADHD medication once daily and 65.7\% (132/201) were taking their primary stimulant medication 7 days a week during the school year for ADHD symptom management. Most $(33.3 \%, 103 / 309)$ of the patients were administered this medication at 7 a.m., whereas 6 p.m. to midnight and 8 a.m. were the second and third most common administration times of day, respectively $(16.5 \%, 51 / 309$ and $13.9 \%, 43 / 309)$. For those patients receiving supplemental medication, most $(23.4 \%, 26 / 111)$ were administered with this medication between 6 p.m. and midnight. Regarding frequency of follow-up with the physician charged with treating patients with ADHD, most (87/201, 43.3\%) were followed up more than three times per year.

When asked to rate the overall severity of treated patient ADHD symptoms on a 10 point scale where 1 indicated no ADHD symptoms and 10 indicated significant ADHD symptoms, respondents reported an average score of 5.45 (Fig. 1). Symptoms of ADHD were regarded as most severe during the Early Morning Routine (6.45) and Evening Homework (6.46) times of day. The average level of overall functional impairment caused by ADHD symptoms during the Early Morning Routine time of day was rated by respondents as 6.09 on a 10 point scale, where 1 indicated mild impairment and 10 indicated severe impairment (Fig. 2). More specifically, 59.7\% (120/201) of caregivers reported overall ADHD symptoms throughout the day as moderate to severe (ADHD symptom score 5-10) and concordantly, 75.6\% (152/201) of parents reported impairment of EMF as moderate to severe (Early Morning Impairment of Functioning score 5-10).

Almost three quarters of respondents reported that easy distractibility $(74.1 \%, 149 / 201)$ and failure to listen $(72.6 \%, 146 / 201)$ 
Table 2. Child/Adolescent Patient Demographic INFORMATION

\begin{tabular}{|c|c|}
\hline & $\mathrm{n}(\%)$ \\
\hline \multicolumn{2}{|l|}{ Gender } \\
\hline Male & 143/201 (71.1) \\
\hline Female & $58 / 201(28.9)$ \\
\hline \multicolumn{2}{|l|}{ Age } \\
\hline $6-12$ & $120 / 201(59.7)$ \\
\hline $13-17$ & $81 / 120(40.3)$ \\
\hline \multicolumn{2}{|l|}{ ADHD subtype } \\
\hline Hyperactive/impulsive & $17 / 201(8.5)$ \\
\hline Inattentive & $26 / 201(12.9)$ \\
\hline Combined & $158 / 201(78.6)$ \\
\hline \multicolumn{2}{|l|}{ Primary ADHD medication } \\
\hline Methylphenidate $\mathrm{HCl}, \mathrm{ER}$ & $61 / 201(30.3)$ \\
\hline $\begin{array}{l}\text { Amphetamine, dextroamphetamine } \\
\text { mixed salts, ER }\end{array}$ & $51 / 201(25.4)$ \\
\hline Methylphenidate $\mathrm{HCl}$, IR & $25 / 201(12.4)$ \\
\hline Lisdexamfetamine dimesylate & $25 / 201(12.4)$ \\
\hline Dexmethylphenidate $\mathrm{HCl}$, IR, and ER & $18 / 201(9.0)$ \\
\hline $\begin{array}{l}\text { Amphetamine, dextroamphetamine } \\
\text { mixed salts, IR }\end{array}$ & $18 / 201(9.0)$ \\
\hline Methylphenidate transdermal & $3 / 201(1.5)$ \\
\hline \multicolumn{2}{|l|}{ Supplemental ADHD medication } \\
\hline Methylphenidate $\mathrm{HCl}, \mathrm{ER}$ & $14 / 201(7.0)$ \\
\hline $\begin{array}{l}\text { Amphetamine, dextroamphetamine } \\
\text { mixed salts, ER }\end{array}$ & $13 / 201(6.5)$ \\
\hline Dexmethylphenidate $\mathrm{HCl}, \mathrm{IR}$, and ER & 4/201 (2.0) \\
\hline Lisdexamfetamine dimesylate & 2/201 (1.0) \\
\hline $\begin{array}{l}\text { Amphetamine, dextroamphetamine } \\
\text { mixed salts, IR }\end{array}$ & $18 / 201(9.0)$ \\
\hline Methylphenidate $\mathrm{HCl}$, IR & $14 / 201(7.0)$ \\
\hline Guanfacine $\mathrm{HCl}$, ER & $7 / 201(3.5)$ \\
\hline Atomoxetine $\mathrm{HCl}$ & 4/201 (2.0) \\
\hline Methylphenidate transdermal & $1 / 201(0.5)$ \\
\hline Other & 4/201 (2.0) \\
\hline None & $120 / 201(59.7)$ \\
\hline
\end{tabular}

Length of time taking primary ADHD medication

3-6 months

$25 / 201(12.4)$

6-12 months

$62 / 201(30.8)$

$1-2$ years

$52 / 201(25.9)$

$>2$ years

$62 / 201(30.8)$

Number of times per day taking primary medication

Once

$135 / 201(67.2)$

Twice

$49 / 201(24.4)$

Three or more

$17 / 201(8.5)$

Time of day taking primary medication $(n=201$ responses with multiple times)

Midnight-5 a.m.

$10 / 309(3.2)$

6 a.m.

$37 / 309(12.0)$

7 a.m.

$103 / 309(33.3)$

8 a.m.

$43 / 309(13.9)$

9 a.m.-noon

$28 / 309(9.1)$

$1-5$ p.m.

$37 / 309(12.0)$

6 p.m.-midnight

$51 / 309(16.5)$

Time of day taking supplemental medication $(n=81$ responses with multiple times)

Midnight-5 a.m

$8 / 111(7.2)$

6 a.m.

7 a.m.

$7 / 111(6.3)$

8 a.m.

9 a.m.-noon

24/111 (21.6)

$13 / 111(11.7)$

$17 / 111(15.3)$

(continued)
TABle 2. (CONTINUED)

\begin{tabular}{lc}
\hline & $\mathrm{n}(\%)$ \\
\hline $1-5$ p.m. & $16 / 111(14.4)$ \\
6 p.m.-midnight & $26 / 111(23.4)$ \\
Days per week taking ADHD medication during the school year \\
7 & $132 / 201(65.7)$ \\
6 & $10 / 201(5.0)$ \\
5 & $50 / 201(24.9)$ \\
4 & $4 / 201(2.0)$ \\
$1-3$ & $5 / 201(2.5)$ \\
Frequency of routine follow-up with ADHD physician \\
Less than once a year \\
Once a year & $11 / 201(5.5)$ \\
Twice a year & $23 / 201(11.4)$ \\
Three times a year & $44 / 201(21.9)$ \\
More than three times a year & $36 / 201(17.9)$ \\
\end{tabular}

ADHD, Attention-deficit/hyperactivity disorder; ER, extended-release; IR, immediate-release.

were the ADHD symptoms that appeared most frequently during Early Morning Routine time of day (Fig. 3). When asked about unwanted behaviors that appeared frequently during the Early Morning Routine time of day, roughly half of the respondents reported that being impulsive/acting without thinking $(49.3 \%$, 99/201) and failure to finish things $(48.8 \%, 98 / 201)$ were the most frequent (Fig. 4). Respondents reported that they often felt overwhelmed and exhausted $(40.8 \%, 82 / 201)$ as a result of patient ADHD symptoms during the Early Morning Routine time of day, and a higher percentage reported sometimes feeling constantly stressed $(46.8 \%, 94 / 201)$, raising their voices more than they wanted to $(43.8 \%, 88 / 201)$, feeling inadequate as a caregiver (43.8\%, 88/201), and punishing his/her child/adolescent more and praising the child/adolescent less $(43.8 \%, 88 / 201$; Fig. 5). Approximately one third $(34.3 \%, 69 / 201)$ of respondents reported that the challenges of the Early Morning Routine, associated with their child's/adolescent's inadequately controlled ADHD symptoms, had a somewhat negative effect on their relationship with the patient and $7.5 \%(15 / 201)$ stated that it had a very negative effect on their relationship with the patient. However, some parents stated that it had a very positive impact $(10 \%, 20 / 201)$, somewhat positive impact $(21.4 \%, 43 / 201)$, or no impact $(26.9 \%, 54 / 201)$ on their overall relationship with the patient. When asked about their satisfaction with their child's/adolescent's current ADHD medication in providing meaningful symptom relief during the Early Morning

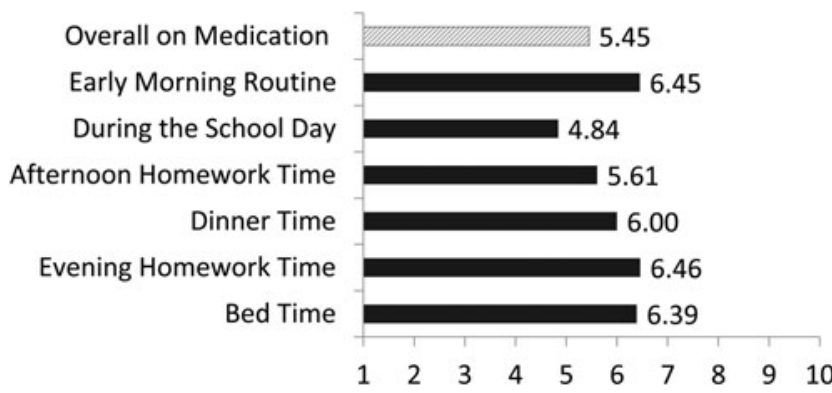

FIG. 1. Attention-deficit/hyperactivity disorder (ADHD) symptom severity, overall and times of day, using a scale of 1-10 where 1 means no ADHD symptoms and 10 means significant ADHD symptoms. 


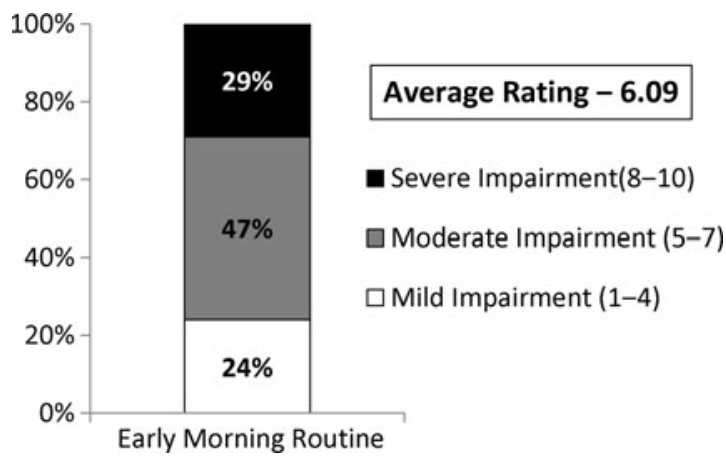

FIG. 2. Overall impairment of functioning from attentiondeficit/hyperactivity disorder symptoms during the Early Morning Routine, using a scale of 1-10 where 1 means "Mild Impairment" and 10 means "Severe Impairment." The average rating of impairment was 6.09 .

Routine time of day, most $(38.8 \%, 78 / 201)$ reported that they were only somewhat satisfied, $10.9 \%(22 / 201)$ were not very satisfied, $3.0 \%(6 / 201)$ were not at all satisfied, $35.8 \%(72 / 201)$ were very satisfied, and $11.4 \%(23 / 201)$ were extremely satisfied. A majority of respondents $(79.1 \%, 159 / 201)$ reported discussing patient EMF impairments with the patient's physician, and almost half (48.3\%, 97/201) reported that they had previously woken up early in order to administer ADHD medication to their child/adolescent in an attempt to help mitigate early morning ADHD symptoms.

\section{Discussion}

The temporal occurrence of inadequately controlled ADHD symptoms, specifically during the Early Morning Routine, has only recently received attention in clinical trials involving stimulant medications. Inadequately controlled early morning ADHD symptoms in stimulant-treated children and adolescents can be attributed to the delayed onset of clinically meaningful symptom control afforded by oral stimulant medications that are dosed subsequent to awakening.

To our knowledge, this is the first quantitative research survey directly assessing parent/caregiver perceptions of: 1) The relative prevalence and severity of inadequately controlled early morning ADHD symptoms, 2) their impact on early morning behaviors and EMF, and 3) their apparent emotional impact on the parent and 4)

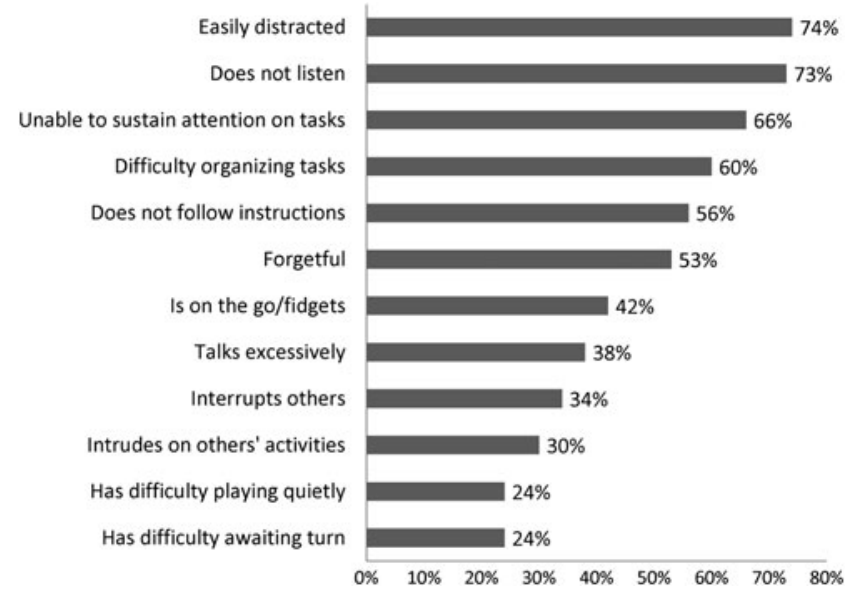

FIG. 3. Attention-deficit/hyperactivity disorder symptoms appearing frequently during the Early Morning Routine.

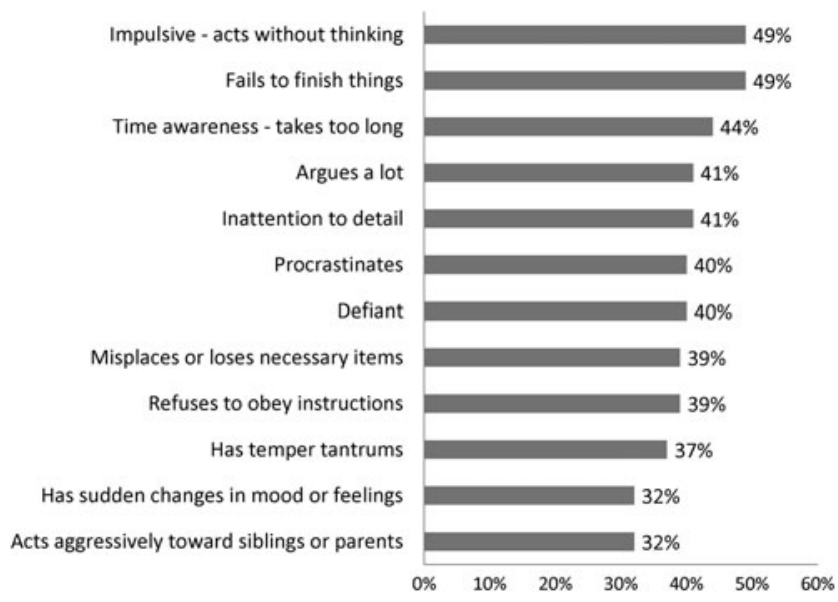

FIG. 4. Unwanted behaviors appearing frequently during the Early Morning Routine.

on the parent-child relationship. As such, this research survey produced several important quantitative findings. First, survey data indicated that caregivers regarded the Early Morning Routine as a period frequently associated with moderate to severe ADHDsymptom-related functional impairments in children and adolescents with ADHD, despite morning administration of stable doses of stimulant medications. Second, caregivers reported a host of negative emotional feelings as a result of their child's inadequately controlled early morning ADHD symptoms. Third, approximately one third of caregivers reported that their child's ADHD symptomrelated functional impairments during the Early Morning Routine contributed to a somewhat negative relationship with their child. Interestingly, caregivers overwhelmingly acknowledged that they had discussed EMF impairments with the child or adolescent's treating physician, and more than half expressed room for improvement regarding satisfaction with the current medication regimen during this time of day.

\section{Limitations}

The current study had several limitations. Survey instruments such as the one developed for the current study may lack some of the specificity of formally validated ADHD assessment tools used to assess symptom severity and functional impact. Furthermore, the surveys were completed through the anonymity of the Internet, were not subject to immediate assessment, and could not offer the opportunity for clarification provided by questionnaires administered during one-on-one research interviews. Survey data are collected in real world circumstances and unlike clinical trials, cannot be fully controlled. The study also did not include an untreated ADHD control group to serve as a comparator, which could have provided some additional insight regarding EMF impairments in children and adolescents with ADHD who were not treated with stimulant medications. It should be noted, however, that parental/ primary caregiver surveys can be an important tool for evaluating the impact of EMF impairments on children and adolescents with ADHD and their caregivers in "real-world" settings, and that such information is difficult to obtain from clinical trials. Additionally, the detailed survey data reported in this study represent an important addition to the sparse clinical literature regarding EMF impairments in children and adolescents with ADHD treated with a stimulant medication, as well as the potential impact of impaired EMF on the parent-child relationship. 


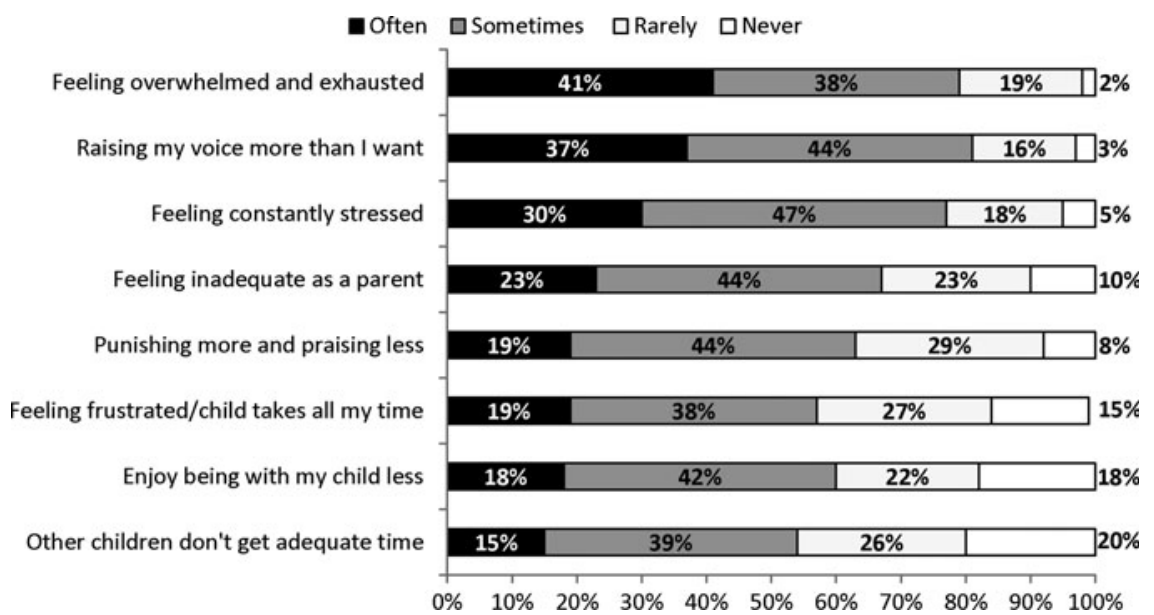

FIG. 5. Caregiver reactions to attention-deficit/hyperactivity disorder symptoms during the Early Morning Routine.

\section{Conclusions}

The issue of ADHD symptom control in the early morning time period is a meaningful issue to parents and caregivers, as almost half $(48.3 \%)$ of caregivers indicated they had woken up early to administer their child's ADHD medication, and $79.1 \%$ of caregivers had previously discussed their child's early morning functional impairments with their doctor. The results from this quantitative research survey study strongly suggest that despite early morning administration of stable doses of existing formulations of stimulant medications, parents/primary caregivers of children and adolescents with ADHD report a high prevalence of inadequately controlled, early morning ADHD symptoms, and the severity of these early morning symptoms and related functional impairments are moderate to severe for a majority of their children. Importantly, they also noted that oftentimes their relationship with their child or adolescent with ADHD was negatively affected by these inadequately managed ADHD symptoms. These results further suggest that pharmacologic management of EMF impairments caused by ADHD symptoms remains a significant unmet need in children and adolescents with ADHD following the morning administration of stimulant medications. Finally, formulation research to explore development of additional stimulant-based treatment options for children and adolescents with ADHD should most likely include a focus on drug delivery mechanisms designed to provide clinically meaningful control of early morning ADHD symptoms and commensurate improvements in overall functioning during the early morning routines of school-age children. Finally, we concur with the observations of Faraone et al. (2015) that, given the clinical and practical impact of ADHD on morning symptoms and functioning, a scale specifically developed and validated to measure morning behaviors impaired by the symptoms of ADHD should be used in future trials of ADHD medications for youth.

\section{Clinical Significance}

Despite improvements in drug delivery systems for ADHD medications, EMF remains an issue in many children and adolescents with ADHD (Whalen et al. 2006). Current stimulant formulations cannot offer meaningful control of ADHD symptoms for at least 1 hour following oral ingestion (Swanson et al. 2003). This study confirms that the early morning routine continues to be a problematic time of the day for children and adolescents with ADHD treated with stimulant medication and their families.

\section{Acknowledgments}

Medical writer William Perlman was engaged to analyze the data, and wrote the first draft of the manuscript. Dr. Sallee provided review, comments, and revisions.

\section{Disclosures}

Dr. Sallee was involved in the development of the survey questions. The data collection was funded by a market research survey for Ironshore Pharmaceuticals \& Development, Inc. Dr. Sallee receives or has received consulting fees from Impax Labs, Ironshore Pharmaceuticals \& Development, Inc., Neos Therapeutics, Otsuka Development and Commercialization, P2D Bioscience, Pfizer, and Skysis LLC. He receives or has received research support from Rhodes Pharma and Shire PLC. He has also served on an advisory board for Astra Zeneca.

\section{References}

American Psychiatric Association: Diagnostic and Statistical Manual of Mental Disorders, 5th ed. Arlington, VA: American Psychiatric Association; 2013.

Antrop I, Roeyers H, De Baecke L: Effects of time of day on classroom behaviour in children with ADHD. Sch Psychol Int 26:29-43, 2005.

Breuer D, Görtz-Dorten A, Rothenberger A, Döpfner M: Assessment of daily profiles of ADHD and ODD symptoms, and symptomatology related to ADHD medication, by parent and teacher ratings. Eur Child Adolesc Psychiatry 20:S289-S96, 2011.

Chang LR, Chiu YN, Wu YY, Gau, SS: Father's parenting and fatherchild relationship among children and adolescents with attentiondeficit/hyperactivity disorder. Compr Psychiatry 54:128-140, 2013.

Coghill D, Soutullo C, d'Aubuisson C, Preuss U, Lindback T, Silverberg M, Buitelaar J: Impact of attention-deficit/hyperactivity disorder on the patient and family: Results from a European survey. Child Adolesc Psychiatry Ment Health 2:31, 2008.

Döpfner M, Görtz-Dorten A, Breuer D, Rothenberger A: An observational study of once-daily modified-release methylphenidate in ADHD: Effectiveness on symptoms and impairment, and safety. Eur Child Adolesc Psychiatry 20:S243-S55, 2011.

Faraone SV, Hammerness PG, Wilens TE: Reliability and validity of the Before-School Functioning Scale in children with ADHD. J Atten Disord 2015 [Epub ahead of print]

Gau SS, Chang JP: Maternal parenting styles and mother-child relationship among adolescents with and without persistent attentiondeficit/hyperactivity disorder. Res Dev Disabil 34:1581-1594, 2013. 
Marketing Research Organization: MRA CODE of Marketing Research Standards, October 2013. Available at http://www.marketingresearch .org/sites/mra.cms.memberfuse.com/mra/files/pdfs/MRA_Code_ 10.15.2013.pdf. Accessed July 7, 2014.

Michelson D, Allen AJ, Busner J, Casat C, Dunn D, Kratochvil, C, Newcorn J, Sallee FR, Sangal RB, Saylor K, West S, Kelsey D, Wernicke J, Trapp NJ, Harder, D: Once-daily atomoxetine treatment for children and adolescents with attention deficit hyperactivity disorder: a randomized, placebo-controlled study. Am J Psychiatry 159:1896-1901, 2002.

Pliszka S, AACAP Work Group on Quality Issues: Practice parameter for the assessment and treatment of children and adolescents with attention-deficit/hyperactivity disorder. J Am Acad Child Adolesc Psychiatry 46:894-921, 2007.

Sangal RB, Owens J, Allen AJ, Sutton V, Schuh K, Kelsey D: Effects of atomoxetine and methylphenidate on sleep in children with ADHD. Sleep 29:1573-1585, 2006.

Shaw M, Hodgkins P, Caci H, Young S, Kahle J, Woods AG, Arnold LE: A systematic review and analysis of long-term outcomes in attention deficit hyperactivity disorder: Effects of treatment and non-treatment. BMC Med 10:99, 2012.

Swanson J, Gupta S, Lam A, Shoulson I, Lerner M, Modi N, Lindemulder E, Wigal S: Development of a new once-a-day formulation of methylphenidate for the treatment of attention-deficit/hyperac- tivity disorder: Proof-of-concept and proof-of-product studies. Arch Gen Psychiatry 60:204-211, 2003.

Whalen CK, Henker B, Jamner LD, Ishikawa SS, Floro JN, Swindle R, Perwien AR, Johnston JA: Toward mapping daily challenges of living with ADHD: maternal and child perspectives using electronic diaries. J Abnorm Child Psychol 34:115-130, 2006.

Wilens TE, Hammerness P, Martelon M, Brodziak K, Utzinger L, Wong, PA: A controlled trial of the methylphenidate transdermal system on before-school functioning in children with attentiondeficit/hyperactivity disorder. J Clin Psychiatry 71:548-556, 2010.

Willcutt EG: The prevalence of DSM-IV attention-deficit/hyperactivity disorder: A meta-analytic review. Neurotherapeutics 9:490-499, 2012.

Wymbs BT, Pelham WE, Jr., Molina BS, Gnagy EM, Wilson TK, Greenhouse JB: Rate and predictors of divorce among parents of youths with ADHD. J Consult Clin Psychol 76:735-744, 2008.

Address correspondence to:

Floyd R. Sallee, $M D, P h D$

UC Department of Psychiatry \& Behavioral Neuroscience 260 Stetson Street; Suite 3200

Cincinnati, $\mathrm{OH} 45219-0559$

E-mail: Floyd.sallee@uc.edu 